Urologe $2020 \cdot 59: 450-460$

https://doi.org/10.1007/s00120-020-01123-x

Online publiziert: 5 . Februar 2020

(c) Der/die Autor(en) 2020

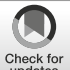

\author{
Jan Herden ${ }^{1,2}$ Edith A. Boedefeld ${ }^{3}$. Lothar Weißbach ${ }^{3}$ \\ 'Medizinische Fakultät und Uniklinik Köln, Klinik für Urologie, Uro-Onkologie, spezielle urologische und \\ roboter-assistierte Chirurgie, Universität zu Köln, Köln, Deutschland \\ ${ }^{2}$ Urologische Partnerschaft Köln, Praxis für Urologie und Andrologie, PAN-Klinik, Köln, Deutschland \\ ${ }^{3}$ Gesundheitsforschung für Männer gGmbH, Berlin, Deutschland
}

\title{
Die nichtinvasive Therapie des organbegrenzten Prostatakarzinoms im Alter - Ergebnisse der HAROW-Studie
}

\section{Einleitung}

Die Ansicht, ein PCa müsse unter allen Umständen und sofort radikal behandelt werden, ist weit verbreitet. Alter, Komorbiditäten, Tumorkriterien und Prostataspezifisches Antigen(PSA)-Verläufe verlangen aber eine individualisierte Behandlung.

Die Früherkennung mittels PSA-Test führt nicht nur zu einer Abnahme fortgeschrittener Stadien, sondern auch zu einer hohen Zahl von „PSA-Kranken“, deren Tumor - würde er nicht diagnostiziert - zu Lebzeiten nie in Erscheinung träte [2]. Fast drei Viertel dieser Karzinome befinden sich zum Zeitpunkt der Diagnose im Stadium cT1 (31\%) bis cT2 (43\%), N0, M0. Bei einem mittleren Erkrankungsalter von 72 Jahren und dem langen Verlauf ist die Prognose mit einem tumorspezifischen 10-Jahres-Überleben von $>90 \%$ so gut wie bei wenigen anderen Tumorentitäten.

Die PCa-Sterblichkeitsrate ist seit mehr als 20 Jahren in Deutschland leicht rückläufig. Vom Höchststand 1994/95 mit >30/100.000 fiel sie bis 2014 auf 19,7; für 2018 werden 19,4 prognostiziert; nur $3,1 \%$ aller Todesfälle sind auf ein $\mathrm{PCa}$ zurückzuführen [2, 3]. Die gleiche Entwicklung wird in den USA beobachtet. Dort sinkt die Mortalität seit 1999, die Indzidenz nimmt ebenfalls signifikant ab: sie fiel von 2008 bis 2014 um 7,6\%, die PCa-Mortalität um 2,2\% [4].

\section{Behandlungsindikation}

Der sich über viele Jahre erstreckende Verlauf der Erkrankung und deren geringes Lebenszeitrisiko relativieren die sofortigen invasiven Interventionen wie radikale Prostatektomie (RP) und Radiotherapie (RT). Männer mit Low-risk-PCa und einer hohen Lebenserwartung gewinnen die Chance, sich durch die weit weniger belastende AS eine unnötige invasive Therapie und deren Folgen zu ersparen, ohne bei evtl. eintretender Progression auf eine kurative Behandlung zu verzichten [5]. Entscheidend sind - neben der individuellen Präferenz - die Belastbarkeit und die Lebenserwartung. Älteren Männern kann bei entsprechenden Voraussetzungen WW empfohlen werden, bei aggressiven Tumoren eine HT.

\section{Langfristige Beobachtung}

Langfristige Beobachtung (WW) ist eine palliative, symptomorientierte Option bei eingeschränkter Lebenserwartung; behandelt wird erst bei krankheitsbedingter Symptomatik. Vorgaben zu PSAKontrollen oder Rebiopsien im Rahmen der Verlaufsbeobachtung sind für WWPatienten per definitionem nicht vorgesehen.

Zwei randomisierte Studien (SPCG-4 und PIVOT) haben WW gegenüber RP geprüft. In der SPCG-4-Studie unterschied sich die tumorspezifische Mortalität nach 18 Jahren nicht signifikant. 


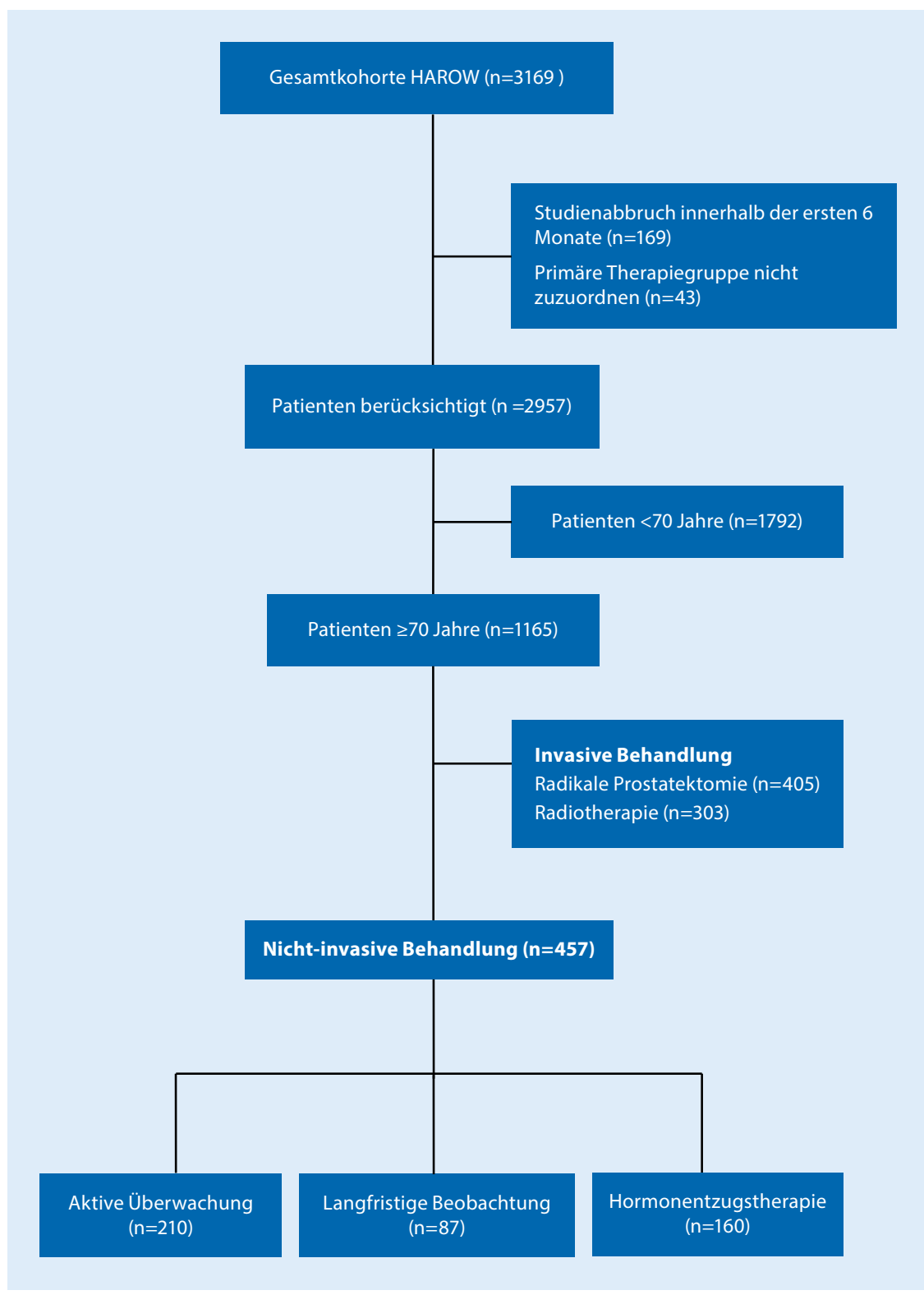

Abb. $1 \Delta$ Patientenselektion innerhalb der HAROW-Studie

Die RP war nur bei Patienten <65 Jahren geringfügig überlegen (tumorspezifische Mortalität 6,8\% vs. $11 \%$ ), obwohl intermediate und High-risk-Tumoren einbezogen waren [6]. Auch in der PIVOT-Studie unterschied sich nach einer medianen Beobachtungszeit von 10 Jahren die tumorspezifische Mortalität nach RP und WW nur gering (5,8 vs. $8,4 \%$; [7]). Die Ergebnisse der Studie sind aufgrund einiger Mängel statistisch nur unzureichend abgesichert. Beide Studien haben den Evidenzlevel $1 \mathrm{~b}$ und finden einen Vorteil der RP gegenüber WW ten und einem PSA-Wert $>50 \mathrm{ng} / \mathrm{ml}$ vorbehalten bleiben [8]. Nach den 15-Jahres-Ergebnissen einer Kohorte mit T1und T2-Tumoren und zusätzlichen Erkrankungen bietet die HT nach 15-jähriger Beobachtung keinen Überlebensvorteil, ist aber mit behandlungsbedingten Nebenwirkungen wie Osteoporose, verminderter Libido und metabolischem Syndrom verbunden. Dem verlängerten progressionsfreien Überleben steht eine uneinheitliche Datenlage bezüglich des Gesamtüberlebens gegenüber [9-11].

\section{Aktive Überwachung}

Während das WW-Konzept in kontrollierten randomisierten Studien (RCT) überprüft war $[6,7]$, wurde AS als eine relativ neue Strategie Anfang der 1990er-Jahre in Kohortenstudien entwickelt. Eine Zeitlang wurden beide Namen zunächst synonym verwendet, dann trennte Parker 2004 Begriffe und Vorgehensweisen. Während für WW das Monitoring als unwichtig erachtet wurde („PSA-testing unimportant, no repeat biopsies“), war für AS die Erkennung der Progression äußerst wichtig, da sie die Intervention veranlasst [12]. Die guten Ergebnisse der AS gehen auf die definierten Eingangskriterien und v. a. auf die konsequenten Kontrollen zurück [13]. AS ist inzwischen eine durch 3 Metanalysen abgesicherte Strategie [14-16].

\section{Die Akzeptanz von Palliation und aktiver Überwachung}

Vor allem die von Klotz et al. erzielten Ergebnisse haben dazu geführt, dass AS in den USA neben RP und RT seit Jahren als Standard gilt $[17,18]$. So erhalten $42 \%$ der Patienten mit organbegrenztem PCa eine AS (oder WW; [18]). Auch in Schweden [5] und Australien [19] ist diese Strategie weit verbreitet - hier werden $74 \%$ bzw. $25 \%$ der Patienten mit neu diagnotiziertem Low-risk-PCa aktiv überwacht. Eine prospektiv randomisierte Studie (ProtecT) belegt, dass sich das krebsspezifische Überleben gegenüber einer sofortigen invasiven Therapie nicht unterscheidet [20]. Auch in Deutschland hat AS 
Urologe 2020 ·59:450-460 https://doi.org/10.1007/s00120-020-01123-x

(c) Der/die Autor(en) 2020

\section{J. Herden · E. A. Boedefeld · L. Weißbach \\ Die nichtinvasive Therapie des organbegrenzten Prostatakarzinoms im Alter - Ergebnisse der HAROW-Studie}

\section{Zusammenfassung}

Hintergrund. Bei Patienten höheren Alters mit einem organbegrenzten Prostatakarzinom (PCa) haben nichtinvasive Therapien wie Active Surveillance (AS), Watchful Waiting (WW) und Hormonentzugstherapie (HT) einen besonderen Stellenwert.

Ziel der Arbeit. Wir überprüfen nichtinvasive Behandlungsformen im Versorgungsalltag an einer Kohorte von Patienten $\geq 70$ Jahren. Material und Methoden. Die Daten stammen aus der HAROW-Studie, mit der die Behandlung des organbegrenzten PCa unter Alltagsbedingungen untersucht wurde. Einziges Eingangskriterium war ein neu diagnostiziertes, organbegrenztes $\mathrm{PCa}$ ( $\leq \mathrm{CT} 2 \mathrm{c}$ ). AS-, WW- und HT-Patienten werden im Hinblick auf die initialen Tumor- und Pati- entencharakteristika, Verlaufsuntersuchungen sowie Therapiewechsel untersucht. Ergebnisse. Von 457 Patienten $\geq 70$ Jahren wählten 210 AS, $160 \mathrm{HT}$ und 87 WW. Die mittleren Beobachtungszeiten waren für AS 6,3 Jahre, WW 7,0 Jahre und HT 7,5 Jahre. ASPatienten waren jünger (73,2 Jahre) als WW(76,0 Jahre) und HT-Patienten (76,9 Jahre) und hatten mit $80 \%$ einen höheren Anteil von Low-risk-Tumoren (WW $31 \%$, HT 19\%). AS-Patienten wechselten die Therapie häufiger (47,1\%, WW 17,2\%, HT 13,1\%), entwickelten seltener Metastasen (1,0\%, WW $4,6 \%$ HT 6,9\%) und hatten mit $94,3 \%$ das beste Gesamtüberleben $(90,8 \%$ WW und $81,9 \% \mathrm{HT}$ ). Innerhalb der ersten 28,4 Monate unterschied sich die mittlere Anzahl der PSA-
Bestimmungen zwischen AS und WW nicht $(6,1$ vs. 5,2; $p=0,09)$; eine Rebiopsie erhielten $37,6 \%$ der AS-, $11,4 \%$ der WW- und $7,0 \%$ der HT-Patienten.

Diskussion. Die Zuordnung zu den kurativen und palliativen Strategien sollte anhand der Patienten- und Tumorcharakteristika definitionsgemäß vorgenommen werden. Für AS-Patienten bieten sich WW oder HT an, wenn wegen Alter oder Komorbiditäten nicht mehr kurativ behandelt werden sollte.

Schlüsselwörter

Lokal begrenztes Prostatakarzinom · Ältere Patienten - Versorgungsforschung · Aktive Überwachung · Langfristige Beobachtung

\section{Noninvasive treatment of organ-confined prostate cancer in elderly patients-results of the HAROW study}

\section{Abstract}

Background. Noninvasive treatment options such as active surveillance (AS), watchful waiting (WW), and hormone deprivation therapy (HT) are particularly important in elderly patients with localized prostate cancer (PCa).

Objectives. We examine the use of these noninvasive treatment options in the everyday care in a cohort of patients $\geq 70$ years old. Materials and methods. In the HAROW study, the treatment of localized PCa under everyday conditions is investigated. The only inclusion criterion was newly diagnosed organ-confined PCa ( $\leq \mathrm{CT} 2 \mathrm{c})$. In AS, WW, and HT patients, we compared initial tumor and patient characteristics, follow-up examinations and changes of therapy.

Results. Of 457 patients $\geq 70$ years, 210 chose AS, $160 \mathrm{HT}$, and $87 \mathrm{WW}$. Observation times were 6.3 years (AS), 7.5 years (HT), and 7.0 years (WW). AS patients (73.2 years) were younger than WW (76.0 years) and HT patients (76.9 years) and had a higher proportion of low-risk tumors (80\%) versus WW (31\%) and HT (19\%). A change of therapy was observed in $47.1 \%$ of AS, $17.2 \%$ of WW and $13.1 \%$ of HT patients. Metastasis occurred in $1.0 \%$ of AS, $4.6 \%$ of WW, and $6.9 \%$ of HT patients. Overall survival was $94.3 \%$ for AS, $90.8 \%$ for WW and $81.9 \%$ for HT. Within the first 28.4 months, the mean number of PSA determinations did not differ between AS and WW (6.1 vs. 5.2; $p=0.09$ ); a rebiopsy was performed in $37.6 \%$ of AS, $11.4 \%$ of WW, and $17 \%$ of HT patients. Conclusions. The allocation to curative and palliative strategies should be made according to patient and tumor characteristics by definition. Palliative procedures may represent concepts in older patients who initially chose a curative AS strategy.

\section{Keywords}

Localized prostate cancer - Older patients . Health service research - Active surveillance . Watchful Waiting ihren Platz in der Leitlinie gefunden und wird zunehmend akzeptiert [8].

Dagegen wird WW oft als tatenloses Warten auf den Tod verstanden. Für Low-risk-Tumoren kann WW bei dem zu erwartendem langsamen Verlauf unter bestimmten Umständen durchaus die angemessene Strategie sein. In Sorge um die möglichen Folgen einer Progression zögert man oft, den Patienten darüber zu informieren, obwohl sich symptomorientierte wirksame Maßnahmen jederzeit anschließen können. Es lohnt sich, für ältere Patienten AS und WW bzw. HT gemeinsam zu betrachten, zumal ein fließender Übergang möglich ist. Das bietet sich besonders für Männer mit Low-/ Intermediate-risk-PCa an, die zunächst AS gewählt haben und später aus Altersoder anderen Gründen eine mögliche radikale Therapie nicht mehr in Betracht ziehen würden. Es ist zu erwarten, dass etwa die Hälfte der für AS geeigneten $\mathrm{Pa}$ tienten eine Altersstufe erreicht, die sie für WW qualifiziert [21].

\section{Patienten und Methode}

Grundlage der vorliegenden Arbeit sind die Daten der 2008 begonnenen und 5 Jahre später beendeten HAROW-Studie (HT, AS, RT, OP [RP], WW), deren Ziel es u. a. war, auf die damals unbekannte Therapieform der AS aufmerksam zu machen, aber auch auf die palliativen Optionen (WW, HT) hinzuweisen. Eingeschlossen wurden neu diagnostizierte, organbegrenzte $\mathrm{PCa}(\leq \mathrm{cT} 2 \mathrm{c})$ ohne Metastasierung. Feste Therapie- 
Tab. 1 Patienten- und Tumorcharakteristika zu Studienbeginn für Patienten $\geq 70$ Jahren unter aktiver Überwachung, langfristiger Beobachtung und Hormonentzugstherapie

\begin{tabular}{|c|c|c|c|c|c|c|c|}
\hline & \multirow{2}{*}{\multicolumn{2}{|c|}{$\begin{array}{l}\text { Aktive Überwachung } \\
(n=210)\end{array}$}} & \multirow{2}{*}{\multicolumn{2}{|c|}{$\begin{array}{l}\text { Langfristige Beobachtung } \\
(n=87)\end{array}$}} & \multirow{2}{*}{\multicolumn{2}{|c|}{$\begin{array}{l}\text { Hormonentzugstherapie } \\
(n=160)\end{array}$}} & \multirow[t]{3}{*}{$p$} \\
\hline & & & & & & & \\
\hline & Median & (IQR) & Median & (IQR) & Median & (IQR) & \\
\hline Alter (Jahre) & 73,2 & $(71,5-75,4)$ & 76,0 & $(73,7-79,8)$ & 76,9 & $(73,9-79,6)$ & $<0,001$ \\
\hline PSA Wert (ng/ml) & 5,5 & $(3,9-7,6)$ & 5,9 & $(3,1-8,8)$ & 9,5 & $(6,1-16,8)$ & $<0,001$ \\
\hline Prostatavolumen (ml) & 40 & $(30-60)$ & 40 & $(25-55)$ & 35 & $(30-50)$ & 0,129 \\
\hline \multirow[t]{2}{*}{ PSA Dichte $\left(\mathrm{ng} / \mathrm{ml}^{2}\right)$} & 0,14 & $(0,08-0,19)$ & 0,16 & $(0,08-0,27)$ & 0,28 & $(0,15-0,52)$ & $<0,001$ \\
\hline & $n$ & (\%) & $n$ & (\%) & $n$ & (\%) & \\
\hline \multicolumn{7}{|l|}{ Tumor Kategorie } & \multirow[t]{6}{*}{$<0,001$} \\
\hline cT1a/b & 53 & $(25,2)$ & 27 & $(31,0)$ & 9 & $(5,6)$ & \\
\hline cT1c & 120 & $(57,1)$ & 38 & $(43,7)$ & 76 & $(47,5)$ & \\
\hline cT2a & 27 & $(12,9)$ & 8 & $(9,2)$ & 10 & $(6,3)$ & \\
\hline $\mathrm{cT} 2 \mathrm{~b}$ & 4 & $(1,9)$ & 3 & $(3,4)$ & 19 & $(11,9)$ & \\
\hline cT2c & 6 & $(2,9)$ & 11 & $(12,6)$ & 46 & $(28,8)$ & \\
\hline \multicolumn{7}{|l|}{ Gleason-Score } & \multirow[t]{5}{*}{$<0,001$} \\
\hline$\leq 6$ & 194 & $(92,3)$ & 44 & $(50,6)$ & 74 & $(46,3)$ & \\
\hline $7 a$ & 11 & $(5,2)$ & 13 & $(14,9)$ & 33 & $(20,6)$ & \\
\hline$\geq 7 \mathrm{~b}$ & 2 & $(1,0)$ & 6 & $(6,9)$ & 51 & $(31,9)$ & \\
\hline n.a. & 3 & $(1,4)$ & 24 & $(27,6)$ & 2 & $(1,3)$ & \\
\hline \multicolumn{7}{|l|}{ D'Amico-Klassifikation } & \multirow[t]{5}{*}{$<0,001$} \\
\hline Niedriges Risiko & 168 & $(80,0)$ & 27 & $(31,0)$ & 31 & $(19,4)$ & \\
\hline Intermediäres Risiko & 30 & $(14,3)$ & 30 & $(34,5)$ & 49 & $(30,6)$ & \\
\hline Hohes Risiko & 8 & $(3,8)$ & 12 & $(13,8)$ & 79 & $(49,4)$ & \\
\hline n.a. & 4 & $(1,9)$ & 18 & $(20,7)$ & 1 & $(0,6)$ & \\
\hline \multicolumn{7}{|c|}{ Anzahl positiver Stanzzylinder pro Biopsie } & \multirow[t]{6}{*}{$<0,001$} \\
\hline $0^{a}$ & 35 & $(16,7)$ & 31 & $(35,6)$ & 8 & $(5,0)$ & \\
\hline 1 & 98 & $(46,7)$ & 22 & $(25,3)$ & 26 & $(16,3)$ & \\
\hline 2 & 58 & $(27,6)$ & 9 & $(10,3)$ & 31 & $(19,4)$ & \\
\hline$\geq 3$ & 11 & $(5,2)$ & 23 & $(26,4)$ & 95 & $(59,3)$ & \\
\hline n.a. & 8 & $(3,8)$ & 2 & $(2,3)$ & 0 & $(0,0)$ & \\
\hline \multicolumn{7}{|l|}{ PSA (ng/ml) } & \multirow[t]{4}{*}{$<0,001$} \\
\hline$\leq 10$ & 190 & $(90,5)$ & 69 & $(79,3)$ & 81 & $(50,9)$ & \\
\hline $10-20$ & 19 & $(9,0)$ & 17 & $(19,5)$ & 44 & $(27,7)$ & \\
\hline$>20$ & 1 & $(0,5)$ & 1 & $(1,1)$ & 34 & $(21,4)$ & \\
\hline \multicolumn{7}{|l|}{ PSA-Dichte (ng/ml/ml) } & \multirow[t]{4}{*}{$<0,001$} \\
\hline$<0,2$ & 148 & $(70,5)$ & 38 & $(43,7)$ & 27 & $(16,9)$ & \\
\hline$\geq 0,2$ & 44 & $(21,0)$ & 28 & $(32,2)$ & 46 & $(28,8)$ & \\
\hline n.a. & 18 & $(8,5)$ & 21 & $(24,1)$ & 87 & $(54,3)$ & \\
\hline \multicolumn{7}{|c|}{ Charlson Comorbidity Index } & \multirow[t]{5}{*}{0,82} \\
\hline 0 & 137 & $(65,2)$ & 52 & $(59,8)$ & 87 & $(54,4)$ & \\
\hline 1 & 42 & $(20,0)$ & 19 & $(21,8)$ & 34 & $(21,3)$ & \\
\hline$\geq 2$ & 27 & $(12,9)$ & 12 & $(13,8)$ & 37 & $(23,1)$ & \\
\hline n.a. & 4 & $(1,9)$ & 4 & $(4,6)$ & 2 & $(1,3)$ & \\
\hline
\end{tabular}




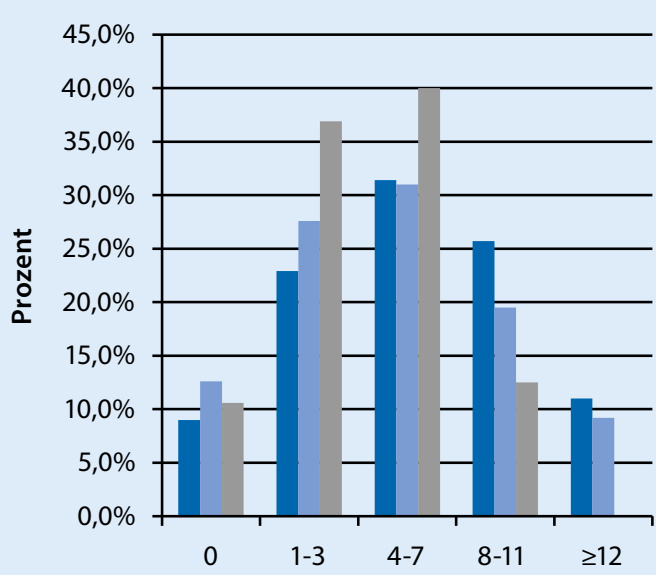

a

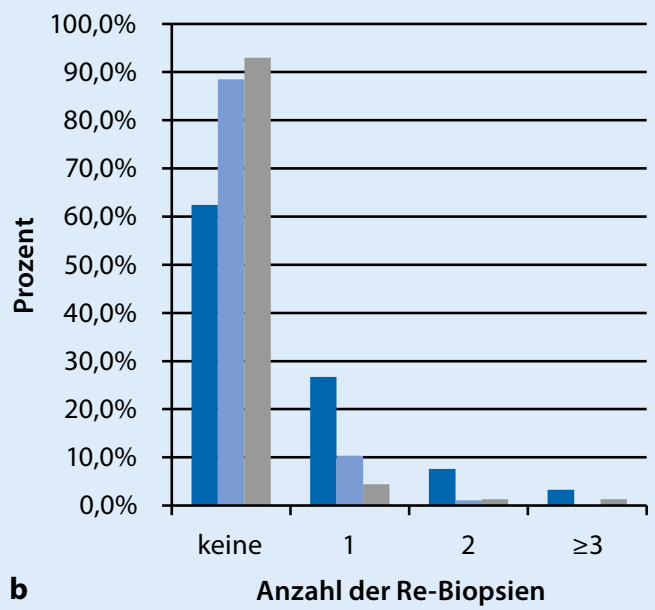

$p<0,001$

- Aktive Überwachung $(n=210)$

Langfristige Beobachtung $(n=87)$

- Hormonentzugstherapie $(n=160)$

$p<0,001$

- Aktive Überwachung $(n=210)$

Langfristige Beobachtung $(\mathrm{n}=87)$

Hormonentzugstherapie $(n=160)$

Abb. $2 \Delta$ a Anzahl der PSA-Bestimmungen (\%) und b Anzahl der Rebiopsien (\%) während der Verlaufskontrollen innerhalb der HAROW-Studie 2008-2013 (mittlere Beobachtungszeit 28,4 Monate)

vorgaben wurden nicht gemacht. Die Therapieentscheidung lag bei den jeweiligen Ärzten/innen bzw. den Patienten. Für AS gab es lediglich Empfehlungen (PSA $\leq 10 \mathrm{ng} / \mathrm{ml}$, Gleason-Score $\leq 6$, PSADichte $\leq 0,2 \mathrm{ng} / \mathrm{ml}^{2}$ und $\leq 2$ positive Biopsien). Weitere Empfehlungen wurden nicht gegeben, da es sich bei WW und HT um etablierte palliative Optionen für Patienten mit einer Lebenserwartung $<10$ Jahren handelte, auf die in früheren Leitlinien eingegangen worden war [22]. Auch die Entscheidung, ob und welche bildgebenden Verfahren eingesetzt wurden, oblag alleine den Studienärzten. Dies war bei der Konzeption der Studie eine bewusste Entscheidung, um die "Real-life-Studienbedingungen“ nicht zu verzerren, da die Studienärzte nicht von Ihrem normalen klinischen Vorgehen abweichen sollten.

HAROW ist also eine prospektive nichtinterventionelle Beobachtungsstudie (NIS) aus dem Bereich der Versorgungsforschung. Die Daten zur Rekrutierung, der Diagnostik (digital-rektale Untersuchung $=$ DRU, PSA, Biopsie) einschließlich des Charlson Comorbidity Index (CCI; [23]) und des Verlaufs der Gesamtkohorte für den Studienzeitraum 2008-2013 mit einer mittleren Beobachtungszeit (mFU) von 28,4 Monaten sind veröffentlicht [1]. Nach Beendigung der Studie wurden einzelne Therapiegruppen gesondert nachbeobachtet, wobei sich diese Nachbeobachtungen auf die wichtigsten Angaben beschränkten: Überleben, Metastasierung und Therapiewechsel (TW). Bei einem TW wurden die gewählte neue Behandlung und der weitere Verlauf erfragt. Eine vollständige Dokumentation aller Angaben konnte durch die gesonderten Nachbefragungen bei $66,2 \%$ der AS-, 67,8 \% der WW- und $76,9 \%$ der HT-Patienten $(p=0,072)$ nach unterschiedlichem $\mathrm{mFU}$ von 6,3 Jahren (AS), 7,0 Jahren (WW) und 7,5 Jahren (HT) erreicht werden. In dieser Arbeit werden jeweils die Subgruppen der $\geq 70$ jährigen Patienten dargestellt.

\section{Statistik}

Die Bewertung wurde mit Version 22 der Statistiksoftware IBM SPSS ${ }^{\circledR}$ durchgeführt. Metrische Variablen wurden mittels univariater Varianzanalyse und dem Kruskal-Wallis-Test bewertet, kategorische Variablen wurden mit dem $\chi^{2-}$ Test oder dem Fisher-Exakt-Test bewertet. Die Analyse des metastasenfreien und Gesamtüberlebens sowie der Zeit bis zu einem TW erfolgte mit der KaplanMeier-Methode und dem Log-RankTest. Die Berechnungen basieren auf dem Signifikanzniveau von $5 \%$.

\section{Ergebnisse}

Von den in die HAROW-Studie eingeschlossenen 3169 Patienten konnten 2957 ausgewertet werden. Von diesen waren $1165(39,4 \%) 70$ Jahre oder älter. In der Gruppe $\geq 70$ Jahre hatten sich $457 \mathrm{Be}$ troffene $(39,2 \%)$ für ein nichtinvasives Vorgehen entschieden: $210(46,0 \%)$ für AS, $87(18,0 \%)$ für WW und $160(35,0 \%)$ für HT (•Abb. 1).

\section{Basisdaten}

Die $\geq 70$-jährigen AS-Patienten waren im Median 73,2 Jahre und im Vergleich zu WW- (76,0 Jahre) und HTPatienten (76,9 Jahre) signifikant jünger $(p \leq 0,001)$. Sie hatten im Median niedrigere PSA-Werte: 5,5 vs. 5,9 vs. $9,5 \mathrm{ng} / \mathrm{ml}(p \leq 0,001)$ und eine niedrigere PSA-Dichte: 0,14 vs. 0,16 vs. $0,28 \mathrm{ng} / \mathrm{ml}^{2}$ $(p \leq 0,001)$. Der Anteil von Tumoren mit Gleason 6 bzw. niedrigem Risiko nach der D'Amico-Klassifikation war in der AS-Gruppe signifikant höher $(92,3 \% / 80 \%)$ als bei WW $(50,6 \% / 31 \%)$ und HT $(46,3 \% / 19,4 \%$; $p \leq 0,001)$. Die 
Hier steht eine Anzeige.

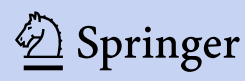




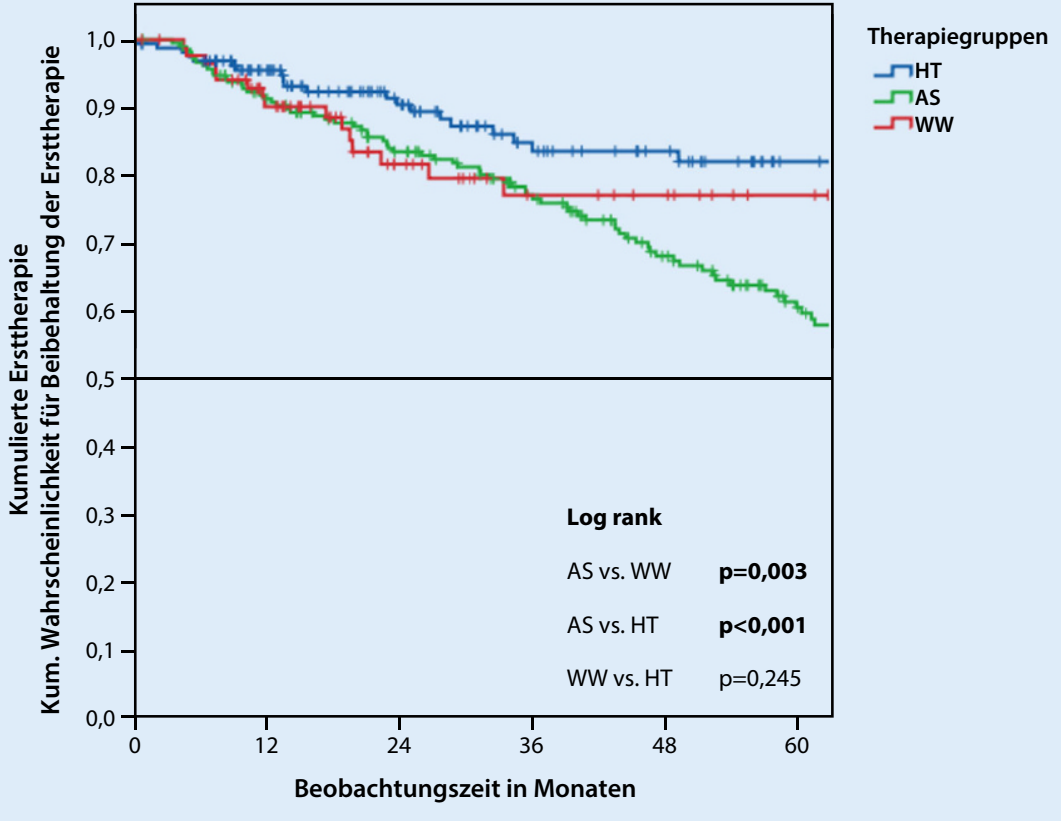

Abb. 3 A Kumulierte Darstellung der Therapiewechsel unter aktiver Überwachung (Active Surveillance, $A S$ ), langfristiger Beobachtung (Watchful Waiting, WW) und Hormonentzugstherapie $(H T)$

initiale Biopsie ergab eine geringere Anzahl von $\geq 3$ positiven Stanzzylindern: $5,2 \%$ vs. $25,4 \%$ vs. $59,3 \%(p \leq 0,001)$. Der Unterschied hinsichtlich des CCI $=0$ war zwischen den drei Gruppen nicht signifikant: $65,2 \%$ vs. $59,8 \%$ vs. $54,7 \%$ $(p=0,82$; Tab. 1$)$.

\section{PSA und Biopsie während des Verlaufs}

Die Daten hierzu wurden nur innerhalb der Rekrutierungszeit der HAROW-Studie ( $m F U ~ 28,4$ Monate) erhoben. Die Häufigkeit der PSA-Bestimmungen betrug in dieser Zeit durchschnittlich 6,1 (AS), 5,2 (WW, $p=0,09$ ) bzw. 3,9 (HT, $p<0,001)$. Vier oder mehr PSA-Bestimmungen während der Beobachtungszeit erhielten $68,1 \%$ (AS), 59,7\% (WW) sowie $52,5 \%$ (HT; - Abb. 2a).

$\mathrm{Zu}$ einer Rebiopsie gelangten 37,6\% der AS-, 11,4\% der WW- und 7,0\% der HT-Patienten (• Abb. 2b). Nach Ende der HAROW-Studie wurden die jährlichen PSA-Bestimmungen sowie die Rebiopsien nicht mehr dokumentiert.

\section{Therapiewechsel}

Die TW im zeitlichen Verlauf der unterschiedlichen Nachbeobachtungszeiten (s. oben) sind in 0 Abb. 3 dargestellt. Nahezu die Hälfte der AS-Patienten $(47,1 \% ; n=99)$ änderte die primär eingeleitete Behandlung, bei WW waren es $17,2 \%(n=15)$, unter HT wechselten die wenigsten Patienten (13,1\%; $n=21)$.

Von den 99 AS-Patienten mit TW ließen sich 55 Patienten $(55,6 \%) \mathrm{ku}$ rativ versorgen $(\mathrm{RP}=27$ oder $\mathrm{RT}=28)$, 31 wechselten $\mathrm{zu}$ WW und 13 zu HT. Somit wurden nach der AS-Phase insgesamt $44,4 \%$ der Patienten in eine Palliation überführt. Die Mehrheit der WW-Wechsler erhielt eine palliative HT $(n=11), 3$ Patienten eine RT und ein Patient ließ sich operieren. Von den HT-Patienten wurden 7 bestrahlt, 7 wechselten zu WW, 6 erhielten eine Chemotherapie und ein Patient wurde mit hochintensivem fokussierten Ultraschall (HIFU) behandelt.

\section{Metastasen und Gesamtüberleben}

Während der Nachbeobachtung entwickelten unter AS 2, unter WW 4 und unter HT 11 Patienten Metastasen, was einem kumulierten metastasenfreien Verlauf von $99,0 \%, 95,4 \%$ und $93,1 \%$ entsprach. Hier zeigten sich signifikante Unterschiede zwischen AS und WW $(p=0,019)$ sowie AS und HT $(p<0,001$; - Abb. 4a). Verstorben waren 12 AS-, 8 WW-, und 29 HT-Patienten. Somit betrug das kumulierte Gesamtüberleben 94,3\% für AS, 90,8 \% für WW und 81,9\% für HT. Hier unterschieden sich nur die AS- und die HT-Gruppe signifikant $(p<0,001$; $\bullet$ Abb. 4b).

\section{Diskussion}

Im klinischen Sprachgebrauch werden die Begriffe „aktive Überwachung“ und „langfristige Beobachtung“ bzw. im angloamerikanischen Sprachgebrauch "Active Surveillance“ und „Watchful Waiting“ oft synonym verwendet. Häufig werden sie auch mit Begriffen wie „expectant management", „conservative management“ oder einfach „observation" umschrieben, welche allerdings keinen Aufschluss darüber geben, ob es sich um einen kurativen oder palliativen Ansatz handelt. Auffällig ist in den Studien SPCG-4 und PIVOT [6, 7], dass der jeweilige WW-Arm nicht eine rein palliative, also symptomorientierte Therapie (Obstruktion der Harnwege, Blutung, Schmerzen und Frakturen) vorsah, sondern eine Form der Beobachtung, die als "kontrolliertes WW“ besser als „kontrolliertes Zuwarten“ bezeichnet werden kann, ohne aber so genannt zu werden. Das wird deutlich an den häufigen Kontrolluntersuchungen und den Wechseln zu kurativ intendierten Therapien. In der HAROW-Studie bestand sogar in der höheren Altersgruppe durchaus die Neigung von Arzt und Patient, den Verlauf unter WW durch häufige PSA-Messungen oder gar Kontrollbiopsien $\mathrm{zu}$ verfolgen, so dass auch wir eher von einem „kontrollierten Zuwarten“ sprechen müssen, auch wenn kurative Interventionen selten waren (• Abb. 2).

Wir haben die Daten der $\geq 70$-jährigen WW- und HT-Patienten denen von AS gegenübergestellt. Das führt wegen der geringeren Selektion - anders als in klinisch kontrollierten Studien (RCT) zu einem versorgungsnahen Abbild. Da- 


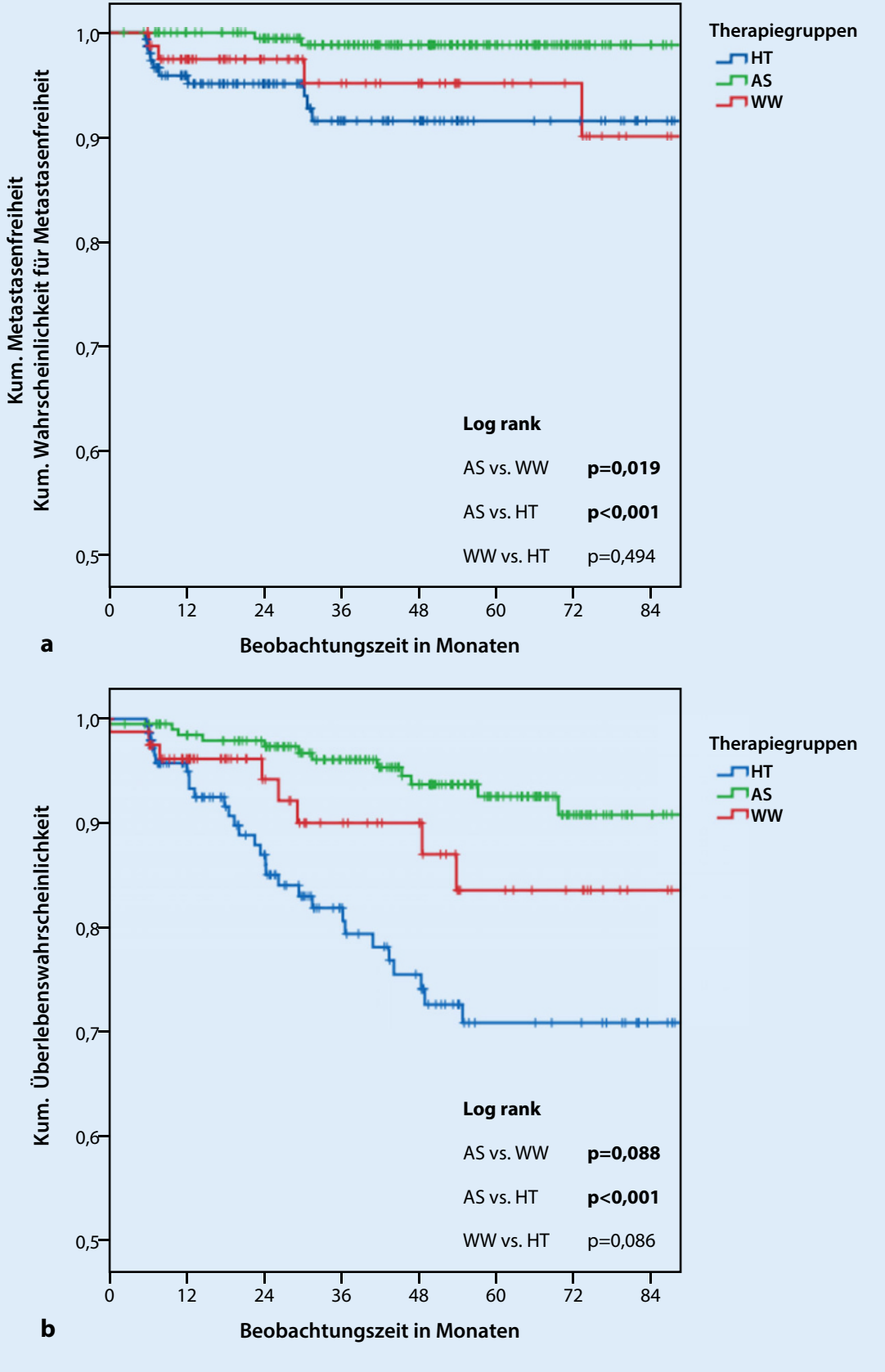

Abb. 4 A a Kumulierte Metastasenfreiheit und b kumuliertes Gesamtüberleben für Patienten unter aktiver Überwachung (Active Surveillance, $A S$ ), langfristiger Beobachtung (WatchfulWaiting, WW) und Hormonentzugstherapie $(H T)$

bei zeigt sich die richtige Zuordnung zu den beiden palliativen Optionen, weil der Wechsel zur Kuration eine Ausnahme blieb. Unter Berücksichtigung der geringen Metastasierung und des guten $\mathrm{Ge}$ samtüberlebens sollten WW und HT vermittelbare Angebote sein, zumal sie die oft ohnehin eingeschränkte Funktionalität der Patienten nicht mindern. Eine kurative Intervention kann sogar wegen der als zur RP oder RT. So ist es zu erklären, dass von 99 AS-Patienten mit einer Therapieänderung $31 \mathrm{zu} \mathrm{WW}$ wechselten. Diese Patienten machen bei der primären Zuordnung die ärztliche Zurückhaltung gegenüber einer Palliation deutlich, indem sie zunächst für eine aufgeschobene kurative Maßnahme vorgesehen wurden.

Die HAROW-Basisdaten der $\geq 70$ Jährigen zeigen die unterschiedliche Zuordnung zur kurativen AS und den palliativen Verfahren WW und HT (- Tab. 1). Die zur Zeit der Studie geltenden Empfehlungen für einen ASEinschluss wurden weitgehend respektiert: $>90 \%$ hatten einen Gleason-Score $\leq 6$, einen PSA-Wert $\leq 10 \mathrm{ng} / \mathrm{ml}$ und $\leq 2$ positive Biopsien.

\section{Wer wechselt die Therapie?}

Auch in der von uns untersuchten höheren Altersgruppe hat jeder zweite Patient AS verlassen, was den Angaben bei normaler Altersverteilung entspricht [15]. Größer ist die „Therapietreue“ bei den primär palliativen Strategien WW und HT. Nur etwa jeder 6. ändert nach 7 Jahren die ursprünglich gewählte Behandlung, wobei die meisten in der Palliation verbleiben. In der richtigen Konsequenz gilt: „einmal palliativ, immer palliativ“.

Von den AS-Wechslern $\geq 70$ wählten $44,4 \%$ eine kurative und 55,6\% eine palliative Therapie. Dies zeigt, dass die Annahme, AS führe zur Kuration insbesondere für ältere Patienten, nicht gilt. Alter, eintretende Morbidität und die Ablehnung einer kurativen Intervention können ein palliatives Angebot nahelegen. Das bedeutet, dass im Alter auch WW und evtl. HT Bestandteil der AS sind. Für einen Patienten, der initial AS gewählt hat, und aufgrund zunehmenden Lebensalters oder neu auftretender Komorbiditäten bei verkürzter Lebenserwartung nicht mehr für eine kurative Therapie in Frage kommt, ist der Übergang zu WW naheliegend. Van Hemelrijk et al. schätzen anhand einer Analyse der nationalen schwedischen Gesundheitsvorsorgeregister, dass $48 \%$ der Männer mit einem „Very-low-risk-PCa“, die mit AS beginnen, im Laufe der Zeit zu WW wechseln. Den Zeitpunkt des Übergangs geben die Autoren im Median mit 
8 Jahren an. 26,5\% der WW-Patienten benötigten eine HT, die übrigen starben an anderen Ursachen [21]. WW ist aus verschiedenen Gründen eine unverzichtbare Strategie, weil Ältere und Alte im Verlauf der Erkrankung nicht mit diagnostischen Maßnahmen belastet werden sollen, da diese häufig ohne therapeutische Konsequenz bleiben.

\section{Kontrolluntersuchungen}

Unter AS sind PSA-Messungen und Rebiopsien unverzichtbar, um den optimalen Zeitpunkt der heilenden Intervention nicht zu verpassen [25]. Hingegen können unter WW PSA-Kontrollen weitgehend unterbleiben, es sei denn, der Tumor ist schlecht differenziert oder es bestehen klinische Zeichen einer Progression. Rebiopsien sind überflüssig. Trotzdem unterschied sich im HAROW-Follow-up die Häufigkeit der PSA-Bestimmungen zwischen AS und WW nicht, sogar Biopsien wurden durchgeführt. Daraus ist zu schließen, dass unter WW psychisch belastende Kontrollen zu häufig sind, während sie in der AS-Gruppe seltener als empfohlen durchgeführt werden. Das entspricht auch der Beobachtung von Loeb et al., nach der $<13 \%$ der AS-Patienten eine Biopsie innerhalb von 2 Jahren erhalten hatten [26]. Innerhalb von 5 Jahren erfüllten nur 11,1 \% die Nachsorgekriterien der Sunnybrook/PRIAS-Kohorten $[13,27]$ bzw. 5,0\% die des Johns Hopkins-Programms [28]. Dass in der HAROW-Studie sogar ältere WW-Patienten eine Rebiopsie erhielten, weist auf die fehlende Abgrenzung gegenüber AS hin.

Thomsen et al. konnten in einer Übersichtsarbeit zeigen, dass etwa die Hälfte der AS-Patienten nach 10 Jahren progredient werden [15]; für viele empfiehlt sich dann die Überleitung in ein WW, weil sie aufgrund ihrer funktionalen Einschränkungen einer invasiven Therapie nicht mehr gewachsen sind. Ohnehin konnten RCT keine bzw. geringe Unterschied im tumorspezifischen Überleben zwischen RP und WW nachweisen [6,7]. Durch die auch von uns beobachtete geringe Metastasierungsrate wird WW für Ältere zu einem Behandlungsangebot, an das bei der initalen Beratung gedacht werden sollte.
Wilt et al. ermutigen Ärzte und Leitliniengruppen dazu, Männer $>65$ Jahre und solche mit Low-risk-Tumoren lediglich zu beobachten, weil durch eine Intervention Schäden besonders im Alter zunehmen, die Mortalität dadurch aber nicht abnimmt [7].

In der HAROW-Studie zeigen sich in der HT-Gruppe ungünstigere Einschlusskriterien mit höheren PSA-Werten und höherer PSA-Dichte; häufiger als bei AS oder WW waren $\geq 3$ Biopsien positiv (-Tab. 1). Diese Daten mögen der Anlass gewesen sein, medikamentös in Form einer HT vorzugehen. Trotzdem sind die Ergebnisse dieser Gruppe am schlechtesten (6,9\% Metastasen, Gesamtüberleben mit 81,9\% am niedrigsten). Dabei ist zu berücksichtigen, dass hier mit 54,7\% die wenigsten $\mathrm{Pa}$ tienten einen CCI von 0 hatten. Die Leitlinie stellt eine Verlängerung des progressionsfreien Überlebens durch die sofortige hormonablative Therapie in Aussicht: „Für Patienten mit einem lokal begrenzten Prostatakarzinom, die eine kurative Therapie oder eine abwartende Haltung ablehnen, ist eine hormonablative Therapie nach ausführlicher Aufklärung eine Option“" [8], weist aber auf die uneinheitliche Datenlage beim Gesamtüberleben hin. Bleibt der Tumor unbehandelt, entspricht das weder der Erwartungshaltung des Patienten noch der Fürsorgeneigung des Arztes. Nach einem systematischen Review lassen sich die progressionsbedingten Komplikationen durch die frühe Androgensuppression vermeiden und das 10Jahres-Überleben mit geringer Signifikanz verbessern [29]. Das gilt jedoch nur für die lokal fortgeschrittenen Tumoren, nicht für die in HAROW untersuchten organbegrenzten Tumoren. Die EORTCStudie 30891 hat gezeigt, dass die frühe Hormonbehandlung Patienten mit aggressivem Tumor vorbehalten sein sollte, da sich die Zeit bis zum Erreichen der Kastrationsresistenz zwischen sofortigem und verzögertem Hormoneinsatz nicht signifikant unterscheidet [10].
Welche Strategie für welchen Patienten?

Zur Beurteilung der Therapiefähigkeit ist eine sorgfältige Einschätzung der vorhandenen körperlichen und mentalen Ressourcen unabdingbar. Diese zeigt, ob der Patient eher für eine AS oder ein WW geeignet ist, da nicht das chronologische Alter, sondern die Lebenserwartung entscheidend ist [30]. Die Einschätzung des biologischen Alters mittels KarnofskyIndex, ECOG- (Eastern Cooperative Oncology Group-) Performance-Status oder CCI liefert zwar prognostische Aussagen über die Lebenserwartung, korreliert aber nur gering mit funktionellem Status und Belastbarkeit. Hilfreich ist in dieser Situation ein geriatrisches Assessment. Die „International Society of Geriatric Oncology“ (SIOG) empfiehlt hier ein dreistufiges Vorgehen, beginnend mit dem G8-Screening [31] und dem Mini$\mathrm{COG}^{\circledR}[32]$. Nur wenn der Patient für eine kurativ intendierte Therapie in Frage kommt, ist AS indiziert. Anderenfalls ist eine palliative Therapie angezeigt [33].

\section{Kosten}

Wenn auch in Deutschland - anders als in den USA - ökonomische Erwägungen bei der Entscheidung im Umgang mit Low-risk-PCa nur selten eine Rolle spielen, so konnten wir am Beispiel der HAROW-Studie auf die erheblichen nach Behandlungsart stark variierenden Folgekosten hinweisen und zeigen, dass die direkten Kosten und die Gesamtkosten aus der Perspektive der Gesellschaft wie auch der Krankenkassen unter WW und HT am niedrigsten sind [34]. In den USA entstehen Medicare bei nicht invasiver Behandlung der 70-Jährigen von der Diagnose bis zur Versorgung der morbiditätsbedingten Folgen einer Behandlung nur $13 \%$ der sonst üblichen Kosten. $\mathrm{Zu}$ bedenken ist dabei, dass fast die Hälfte der ausgewerteten Daten von Patienten mit Gleason-6-Tumoren entsprach [35].

\section{Limitationen}

Die HAROW-Studie ist die einzige deutsche Versorgungsforschungsstudie zum PCa mit einem Vergleich kurativer und 
palliativer Behandlungen. Das gilt naturgemäß auch für Patienten > 70 Jahren Die Aussagen werden limitiert durch die für weiterführende Schlussfolgerungen begrenzten Beobachtungszeiten von maximal 7,5 Jahren sowie die begrenzte Datenqualität, die ein grundsätzliches Problem der Versorgungsforschung im Vergleich $\mathrm{zu}$ RCT ist. Eine weitere Limitation sind die relativ hohen „Drop-out-Raten“, die v. a. durch die fehlenden Aussagen zum tumorspezifischen Überleben entstehen. Auf Nachfragen über die Vertrauensstelle (VS Ltg.) Gemeinsames Krebsregister der Länder Berlin, Brandenburg, Mecklenburg-Vorpommern, Sachsen-Anhalt und der Freistaaten Sachsen und Thüringen (GKR) wurde uns mitgeteilt, dass nicht die Voraussetzungen bestünden, um eine Auskunft von dieser Einrichtung über die verstorbenen Patienten zu erhalten.

\section{Fazit für die Praxis}

- Patienten $\geq 70$ Jahre mit einem organbegrenzten Prostatakarzinom (PCa) sollte auch eine nichtinvasive Behandlung angeboten werden.

- Die Zuordnung zu den kurativen und palliativen Strategien sollte anhand der Patienten- und Tumorcharakteristika definitionsgemäß vorgenommen werden.

- Von Seiten älterer Patienten ist ein geriatrisches Assessment hilfreich, wenn zwischen kurativer oder palliativer Option entschieden werden soll.

- Im Versorgungalltag ist hinsichtlich der Auswahlkriterien und einer verzögerten Therapieoption zwischen AS (Active Surveillance) und WW (Watchful Waiting) zu unterscheiden.

- Häufige PSA-Messungen (prostataspezifisches Antigen) oder gar Biopsien sind während der Palliation nicht notwendig.

- AS-Patienten sollten bei sich entwickelnder Komorbidität und Einschränkung der Funktionalität nach WW oder Hormonentzugstherapie (HT) wechseln.

- Eine palliative HT sollte für ältere Patienten mit einem organbegrenzten PCa eher eine Ausnahme bleiben.

\section{Korrespondenzadresse}

PD Dr. med. Jan Herden

Medizinische Fakultät und Uniklinik Köln, Klinik für Urologie, Uro-Onkologie, spezielle urologische und roboter-assistierte Chirurgie, Universität zu Köln

Kerpener Straße 62, 50937 Köln, Deutschland jan.herden@uk-koeln.de

Förderung. Die HAROW-Studie wurde von der Stiftung Männergesundheit initiiert und durchgeführt sowie von Gazprom Germania finanziell unterstützt.

Funding. Open Access funding provided by Projekt DEAL.

\section{Einhaltung ethischer Richtlinien}

Interessenkonflikt. L. Weißbach wird honoriert für Beratungstätigkeit bei der AOK (Wido). Studienunterstützung (Drittmittel) wurde ihm zuteil von der Firma Gazprom Germania. J. Herden und E.A. Boedefeld geben an, dass kein Interessenkonflikt besteht.

Alle beschriebenen Untersuchungen erfolgten mit Zustimmung der Ethik-Kommission der Bayrischen Landesärztekammer (Nr. 08012), im Einklang mit nationalem Recht sowie der Deklaration von Helsinki von 1975 (in der aktuellen Fassung). Von allen beteiligten Patienten liegt eine Einverständniserklärung vor.

Open Access. Dieser Artikel wird unter der Creative Commons Namensnennung 4.0 International Lizenz veröffentlicht, welche die Nutzung, Vervielfältigung, Bearbeitung, Verbreitung und Wiedergabe in jeglichem Medium und Format erlaubt, sofern Sie den/die ursprünglichen Autor(en) und die Quelle ordnungsgemäßnennen, einen Link zur Creative Commons Lizenz beifügen und angeben, ob Änderungen vorgenommen wurden.

Die in diesem Artikel enthaltenen Bilder und sonstiges Drittmaterial unterliegen ebenfalls der genannten Creative Commons Lizenz, sofern sich aus der Abbildungslegende nichts anderes ergibt. Sofern das betreffende Material nicht unter der genannten Creative Commons Lizenz steht und die betreffende Handlung nicht nach gesetzlichen Vorschriften erlaubt ist, ist für die oben aufgeführten Weiterverwendungen des Materials die Einwilligung des jeweiligen Rechteinhabers einzuholen.

Weitere Details zur Lizenz entnehmen Sie bitte der Lizenzinformation auf http://creativecommons.org/ licenses/by/4.0/deed.de.

\section{Literatur}

1. Herden J, Ansmann L, Ernstmann N et al (2016) The treatment of localized prostate cancer in everyday practice in Germany. Dtsch Arztebl Int 113:329-336

2. Robert Koch Institut (2017) Prostata C61. https://www.krebsdaten.de/Krebs/DE/ Content/Publikationen/Krebs_in_Deutschland/ kid_2017/kid_2017_c61_prostata.pdf? blob=publicationFile. Zugegriffen:4.Okt. 2019
3. Haberland J, Wolf U (2015) Trendanalysen zur Inzidenz und Mortalität an Krebs in Deutschland seit 1970. GMS Med Inform Biom Epidemiol 11:Doc3

4. Cronin KA, Lake AJ, Scott S et al (2018) Annual report to the nation on the status of cancer, part I: national cancer statistics. Cancer 124:2785-2800

5. Loeb S, Folkvaljon Y, Curnyn C et al (2017) Uptake of active surveillance for very-low-risk prostate cancer in Sweden. JAMA Oncol 3:1393-1139

6. Bill-Axelson A, Holmberg L, Garmo H et al (2014) Radical prostatectomy or watchful waiting in early prostate cancer. N Engl J Med 370:932-942

7. Wilt TJ, Jones KM, Barry MJ et al (2017) Followup of prostatectomy versus observation for early prostate cancer. N Engl J Med 377:132-142

8. Leitlinienprogramm Onkologie (2019) Leitlinie Prostatakarzinom. http://www. leitlinienprogramm-onkologie.de/leitlinien/ prostatakarzinom/.Zugegriffen:4.Okt. 2019

9. Ip S, Dahabreh IJ, Chung M et al (2011) An evidence review of active surveillance in men with localized prostate cancer. Evid Rep Technol Assess 204:1-341

10. Studer UE, Whelan $P$, Wimpissinger F, Casselman J, de Reijke TM, Knonagel H, Loidl W, Isorna S, Sundaram SK, Collette L (2014) Differences in time to disease progression do not predict for cancerspecific survival in patients receiving immediate or deferred androgen-deprivation therapy for prostate cancer:final results of EORTC randomized trial 30891 with 12 years of follow-up. Eur Urol 66:829-838

11. Lu-Yao GL, Albertsen PC, Moore DF, Shih W, Lin Y, DiPaola RS, Yao SL (2014) Fifteen-year survival outcomes following primary androgendeprivation therapy for localized prostate cancer. JAMA Intern Med 174:1460-1467

12. Parker C (2004) Active surveillance: towards a new paradigm in the management of early prostate cancer. Lancet Oncol 5:101-106

13. Klotz L, Vesprini D, Sethukavalan P et al (2015) Long-term follow-up of a large active surveillance cohort of patients with prostate cancer. JClin Oncol 33:272-277

14. Dall'Era MA, Albertsen PC, Bangma $C$ et al (2012) Active surveillance for prostate cancer: a systematic review of the literature. Eur Urol 62:976-983

15. Thomsen FB, Brasso K, Klotz LH et al (2014) Active surveillance for clinically localized prostate cancer-a systematic review. J Surg Oncol 109:830-835

16. Simpkin AJ, Tilling $K$, Martin RM et al (2015) Systematic review and meta-analysis of factors determining change to radical treatment in active surveillance for localized prostate cancer. Eur Urol 67:993-1005

17. Womble PR, Montie JE, Ye Z et al (2015) Contemporary use of initial active surveillance among men in michigan with low-risk prostate cancer. Eur Urol 67:44-50

18. Mahal BA, Butler S, Franco I et al (2019) Use of active surveillance or watchful waiting for low-risk prostate cancer and management trends across risk groups in the United States, 2010-2015. JAMA 321:704-706

19. Weerakoon M, Papa N, Lawrentschuk N et al (2015) The current use of active surveillance in an Australian cohort of men: a pattern of care analysis from the victorian prostate cancer registry. BJU Int 115(5):50-56

20. Hamdy FC, Donovan JL, Lane JA et al (2016) 10 -year outcomes after monitoring, surgery, or 
radiotherapy for localized prostate cancer. $\mathrm{N}$ Engl J Med 375:1415-1424

21. Van Hemelrijck $M$, Garmo $H$, Lindhagen $L$ et al (2017) Quantifying the transition from active surveillance to watchful waiting among men with very low-risk prostate cancer. Eur Urol 72:534-541

22. Heidenreich A, Aus G, Bolla M et al (2008) EAU guidelines on prostate cancer. Eur Urol 53:68-80

23. Charlson ME, Pompei $P$, Ales $K L$, MacKenzie CR (1987) A new method of classifying prognostic comorbidity in longitudinal studies: development and validation. JChronic Dis 40:373-383

24. Ernstmann N, Jaeger J, Kowalski C, Pfaff $\mathrm{H}$, Weißbach L (2013) Der ältere Prostatakrebspatient-Information und Einbezug in die Therapieentscheidung. Urologe A 52:847-852

25. Bokhorst LP, Valdagni R, Rannikko A et al (2016) A decade of active surveillance in the PRIAS study: an update and evaluation of the criteria used to recommend a switch to active treatment. Eur Urol 70:954-960

26. Loeb S, Walter D, Curnyn C et al (2016) How active is active surveillance? Intensity follow-up during active surveillance for prostate cancer in the United States. JUrol 196:721-726

27. Bul M, Zhu X, Valdagni Ret al (2013) Active surveillance for low-risk prostate cancer worldwide: the PRIAS study. Eur Urol 63:597-603
28. Tosoian JJ, Trock BJ, Landis P et al (2011) Active surveillance program for prostate cancer: an update of the Johns Hopkins experience. J Clin Oncol 29:2185-2190

29. Nair B, Wilt T, MacDonald R, Rutks I (2002) Early versus deferred androgen suppression in the treatment of advanced prostatic cancer. Cochrane Database Syst Rev 1:CD3506

30. Fröhner M, Wirth M (2017) Therapie des Prostatakarzinoms. Profitieren auch alte Patienten? Allgemeinarzt 39:16-18

31. Soubeyran P, Bellera C, Goyard J et al (2014) Screening for vulnerability in older cancer patients: the ONCODAGE prospective multicenter cohort study. PLoSOne 9:e115060

32. Borson S, Scanlan JM, Chen P, Ganguli M (2003) The mini-cog as a screen for dementia: validation in a population-based sample. J Am Geriatr Soc 51:1451-1454

33. Droz JP, Albrand G, Gillessen S et al (2017) Management of prostate cancer in elderly patients: recommendations of a task force of the international society of geriatric oncology. Eur Urol 72:521-531

34. Reinhold T, Dornquast C, Börgermann C, Weißbach $L$ (2016) Behandlungskosten des lokal begrenzten Prostatakarzinoms in Deutschland.
Ökonomische Ergebnisse der HAROW-Beobachtungsstudie. Urologe A 55:1573-1585

35. Trogdon JG, Falchook AD, Basak R et al (2018) Total medicare costs associated with diagnosis and treatment of prostate cancer in elderly men. JAMA Oncol 5:60-66

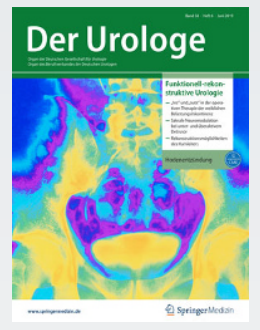

Auf SpringerMedizin.de erhalten Sie Zugang zu allen elektronisch verfügbaren Ausgaben und dem CME-Angebot Ihrer Zeitschrift - unabhängig davon, seit wann Sie Der Urologe abonniert haben. Außerdem können Sie die Zeitschrift mit dem E-Paper auch bequem auf Ihrem Tablet lesen.

So einfach erhalten Sie Zugang zum Online Archiv Registrieren Sie sich einmalig auf www.springermedizin.de/register

Geben Sie dabei Ihre Einheitliche Fortbildungsnummer (EFN) an

- Ihr Benutzername entspricht Ihrer E-Mail-Adresse, Ihr Passwort können Sie frei wählen und später jederzeit unter "Mein Profil“ ändern.

- Falls Sie bereits ein (Print-) Abonnement bei uns haben, geben Sie bei der Registrierung die Lieferadresse Ihrer Zeitschrift an. Damit wird Ihr Abo-Zugang auf springermedizin.de freigeschaltet.
Sind Sie bereits bei SpringerMedizin.de registriert? Dann wird Ihr Zeitschriftenabonnement automatisch Ihrem Online-Nutzerkonto hinzugefügt. Sollten die Angaben Ihres Online-Accounts nicht eindeutig mit den Angaben Ihres Zeitschriften-Abonnements übereinstimmen, kann die Zuordnung nicht sicher erfolgen. In diesem Fall und bei allen anderen Fragen zum Online-Zugang kontaktieren Sie bitte unseren Kundenservice unter: Kundenservice@springermedizin.de

Telefonisch erreichen Sie die Hotline montags bis freitags von 9.00 bis 17.00 Uhr kostenfrei unter 0800-77 80777 sowie gebührenpflichtig aus dem Ausland unter«+49 30884293600 